\title{
The options for the UK leaving the coronavirus lockdown of 2020
}

\section{Philip Thomas*}

\author{
Safety Systems Research Centre, South West Nuclear Hub, Faculty of Engineering, \\ University of Bristol, Queen's Building, University Walk, Bristol BS8 1TR, UK
}

A computer simulation of the Covid-19 epidemic has been updated with data that became available on 14 May 2020. The model parameters have been adjusted (i) to provide the best match to data from Public Health England on daily new labconfirmed infections to 30 April 2020 and (ii) to ensure that the model reproduces the Office of National Statistics central estimate of the average number of recently infected people in England in the week before and the week after 3 May 2020. The new information allows outline guidance to be given for the first time on the approximate consequences of each of the four main options for the UK coming out of lockdown in the absence of an effective vaccine. The options may be ranked based on minimizing the sum of deaths due to Covid-19 and those due to national impoverishment. The Government's currently declared aim of keeping the basic reproduction number below 1.0 emerges as the worst in terms of life preservation.

Keywords: coronavirus, Covid-19, economic challenge, J-value, lockdown, lockdown exit strategy, multi-cohort epidemic model

\section{Introduction}

The coronavirus ${ }^{1}$ pandemic originated in China in the autumn of 2019 but had spread to other countries by December of that year or January of the next. As cases and deaths mounted, many nations, including the USA and most of Europe, decided to put their populations into lockdown.

* E-mail: philip.thomas@bristol.ac.uk; research website: www.jvalue.co.uk

1 The World Health Organization (WHO) has given the new strain of coronavirus the name SARS-Cov-19 (severe acute respiratory syndrome coronavirus 2019). The virus causes the disease COVID-19, an acronym coined by the WHO from "coronavirus disease 2019". This paper adopts the convention used by The Times and other UK newspapers in capitalizing only the first letter of the name. Only one coronavirus is considered in the paper, thus allowing "coronavirus" to be used as a synonym for SARS-Cov-19. 
The confinement instituted in the UK became effective on the evening of 23 March 2020, when the hospitality and entertainment industries were told they needed to close their doors. Schools were instructed to suspend teaching soon after and universities followed suit. Most types of shop were instructed to shut, although not grocery supermarkets nor pharmacies. Employees in all industries were expected to work at home if at all possible. People were told to stay in their homes except for work, medical reasons, buying food or taking exercise once a day locally. Visits to second homes and overnight stays in general were forbidden except in a limited set of circumstances.

Irrespective of whether their industry was officially sanctioned to continue, many businesses decided to suspend most or all of their activities where home working was not feasible. Construction was permitted by the Government, but some regional authorities were hostile to the idea and many construction firms closed. Building companies were, in any case, running out of materials as a result of supply chains being disrupted. For example, builders' merchants were finding it difficult to source replacement products and materials from manufacturers who were closing down for the duration.

The lockdown, as originally announced, was expected to last 3 weeks, subject to review. But it was extended at the end of the first period for a further three weeks and later for longer still. The position in mid May 2020 was that people in all industries except general ("nonessential") retail, hospitality and entertainment were being encouraged to return to work, as from 13 May 2020. That date also marked the lifting of restrictions on travel away from home in England (although not in Wales, Scotland and Northern Ireland). It was intended that schools should begin to open from 1 June 2020 (for reception, year 1 and year 6 classes) and hospitality in July 2020. The return to work would be accompanied by industry-specific social distancing ${ }^{2}$ measures, such as positioning perspex shields between workers.

The reopening of schools was resisted strongly by the teaching unions. There was also a reluctance to return to work amongst workers more generally, many of whom were either receiving their normal salary or being furloughed. Under the furlough scheme, $80 \%$ of an employee's income up to $£ 2,500$ per month was provided by the Government until 1 August 2020 , with a further extension to the end of October 2020 planned during which the employer would need to pay part of the bill. On the other hand, many in England, furloughed or otherwise, took advantage of the newly granted freedom to travel and flocked to beaches to enjoy the exceptionally warm and sunny weather that characterized most of the lockdown period in the UK.

A feature of the Government's message in lifting the lockdown restrictions was its insistence that the effective reproduction number, or " $R$-rate", $R$ should be kept below 1.0. It said it would reintroduce restrictions should $R$, that is to say the number of people infected by the average person with Covid-19, rise above unity. The consequences of such a policy will be assessed.

2 "Social distancing" is the phrase used to describe a set of measures to reduce the likelihood of personto-person spread of coronavirus, ranging from the use of face masks, through staying a prescribed distance away from someone outside one's own household to ordering shops to close. See, for example, Metropolitan Police, 2020, Social distancing rules during coronavirus in England, https:// www.met.police.uk/advice/advice-and-information/c19/coronavirus-covid-19/coronavirus-socialdistancing-rules-england/ 
The $R$-rate is a composite variable, equal to the basic reproduction number, $R_{0}$ multiplied by the fraction, $f_{s}$, of the population that is still susceptible to the virus: $R=f_{s} R_{0}$. In the early stages of the epidemic, $f_{s}=\sim 1$ and so $R=\sim R_{0}$. This approximation is used in the first of the strategies considered.

This paper will explore the four main options for leaving lockdown in the absence of a vaccine, which are:

1. Keep the basic reproduction number, $R_{0}$, below 1.0 ;

2. Move out of lockdown as quickly as possible without overstraining the health services;

3. Move out completely in 2020 while minimizing Covid-19 cases; and

4. Move out as fast as possible.

It is assumed that the epidemiological parameters characterizing the virus will stay constant from 2020 to 2024, unaffected by any possible future mutation. Full allowance is made for a possible 2 nd or 3 rd wave.

Recently documented methods ${ }^{3,4}$ will be used to examine each strategy for its associated total loss of life, both from the coronavirus directly and as a result of national impoverishment should a long and deep recession ensue as a result of the lockdown.

The layout of the paper is as follows. After a first section that sets the problem in context and specifies the leaving options, $\$ 2$ will explain the matching of the two-cohort epidemic model to the empirical data. $\S 3$ will provide a mathematical characterization of the exit process. $\S 4$ will discuss how both Covid-19 and national impoverishment can cause loss of life. $\S 5$ explains how the loss of life expectancy denominated in average UK human lives may be found, both from the deaths caused directly by Covid-19 and from national impoverishment. \$6 will give comparative results for sensitivity studies based on a slightly different set of parameters that give as good a match to the measured data as the original set. $\$ 7$ consists of a discussion, while conclusions are given in $\S 8$. Appendix A explains the concept of "social distancing effectiveness" and its relation to the basic reproduction number $R_{0}$.

\section{Fitting the two-cohort model to the recorded data}

The two-cohort model described in Appendix A of a previous paper ${ }^{3}$ was used to model the Covid-19 epidemic. People in cohort 1 are expected to experience significant symptoms if they contract SARS-CoV-2. It is assumed that anyone in this group falling ill will be tested for the active virus and found positive. Some of these positive cases (but not all) will be admitted to hospital. Meanwhile members of cohort 2 will experience less severe symptoms or else be asymptomatic and will never be subjected to a viral test.

The lockdown is taken to have come into force at 00:01 h on 24 March 2020, a date characterized in the equations as $t_{L}$, subscript $L$ signifying "lockdown". It is assumed that the general public became gradually more proficient at conforming over the next 30 days. This renders the basic reproduction number $R_{0 i}, i=1,2$ (corresponding to the two cohorts) time-varying for each of the two

3 Thomas, P. J-value assessment of how best to combat COVID-19. Nanotechnol. Perceptions 16 (2020) 16-40.

4 Thomas, P. The length and severity of the coronavirus recession estimated from the dynamics of relaxing lockdown. Nanotechnol. Perceptions 16 (2020) 100-129. 
cohorts, decreasing during the lockdown according to the following expression:

$$
R_{0 i}(t)=\left\{\begin{array}{ll}
R_{0 i}(0) & t<t_{L} \\
\left(1-\frac{f_{\Delta R 0}}{30}\left(t-t_{L}\right)\right) R_{0 i}(0) & t_{L} \leq t<t_{L}+30 \\
\left(1-f_{\Delta R 0}\right) R_{0 i}(0) & t \geq t_{L}+30
\end{array} \quad \text { for } i=1,2\right.
$$

where $f_{\Delta R 0}$ is the fractional decrease in the two basic reproduction numbers brought about by lockdown, which is assumed to take 30 days to complete. A derivation of this equation is given in Appendix A, Section A.1.

Seven constants used in the model were adjusted so as to provide the best fit to data gathered on the epidemic to date. Adjustments were made to $R_{01}(0), R_{02}(0), f_{\Delta R 0}, \tau_{\text {inf }, 1}, \tau_{\text {inf }, 2}, \theta_{1}$ and $n_{2}(0)$, where $R_{01}(0), R_{02}(0)$ and $f_{\Delta R 0}$ have been defined above, while $\tau_{\text {inf }, 1}$ is the average time between receiving and passing on infection in cohort $1, \tau_{\text {inf, }}$ is the average time between reception and transmission of infection in cohort $2, \theta_{1}$ is the fraction of the population who are members of the vulnerable cohort 1 (with the remaining $\theta_{2}=1-\theta_{1}$ belonging to cohort 2) and $n_{2}(0)$ is the number of people with an active infection at the start of the simulation, 30 January 2020. The purpose of the parameter adjustments was to minimize the sum of the squared errors and so find the best match to:

(i) daily new lab-confirmed infections 5 to 30 April 2020; and

(ii) the central estimate ${ }^{6}$ of the average number of recently infected people in England in the weeks before and after 3 May 2020.

The optimal set of parameters is given in Table 1 .

Table 1. Epidemic parameter set for base cases of $\S 4$.

\begin{tabular}{lcc}
\hline & Cohort 1 & Cohort 2 \\
\hline Cohort index, $i$ & 1 & 2 \\
Fraction of population in cohort, $\theta_{i}$ & 0.116 & 0.884 \\
Basic reproduction number, $R_{0 i}$ & 2.53 & 1.868 \\
Average time between infections, $\tau_{\text {inf, } i}$ (days) & 8.82 & 8.25 \\
Number of people with active infections on 30 January & 1 & 167 \\
$2020, n_{i}(0)$ & & Applicable to both cohorts
\end{tabular}

$2020, n_{i}(0)$

Applicable to both cohorts

Combined basic reproduction number before lockdown, $R_{0}$ 1.94

Population ("herd") immunity fraction

$49 \%$

Fractional decrease in the two basic reproduction numbers

after 30 days of lockdown, $f_{\Delta R 0}$

0.619

Combined basic reproduction number after 30 days of

lockdown

0.74

Overall fatality rate after infection

0.0057

Root mean squared error between daily case numbers to

30 April 2020

388

5 GOV.UK. Coronavirus (COVID-19) in the UK, https://coronavirus.data.gov.uk/ (accessed 19 May 2020).

${ }^{6}$ Office for National Statistics. Coronavirus (COVID-19) Infection Survey pilot: England (14 May 2020). 
The ONS survey figure for the number of active infections outside hospitals is reproduced to 1 part in 100,000. Meanwhile the match to daily new infections is given in Figure 1. The root mean squared error (RMSE) is 388 cases, higher than the corresponding figure of 212 reported previously ${ }^{3}$ when the end date was taken to be 10 April as opposed to 30 April 2020. The higher RMSE is brought about by greater exposure to the noisier data evident from the beginning of April onwards. This larger temporal variation appears to be mainly a weekend effect: 12 out of 15 data points falling below the fitted line from 28 March 2020 onwards occurred on either a Saturday or a Sunday.

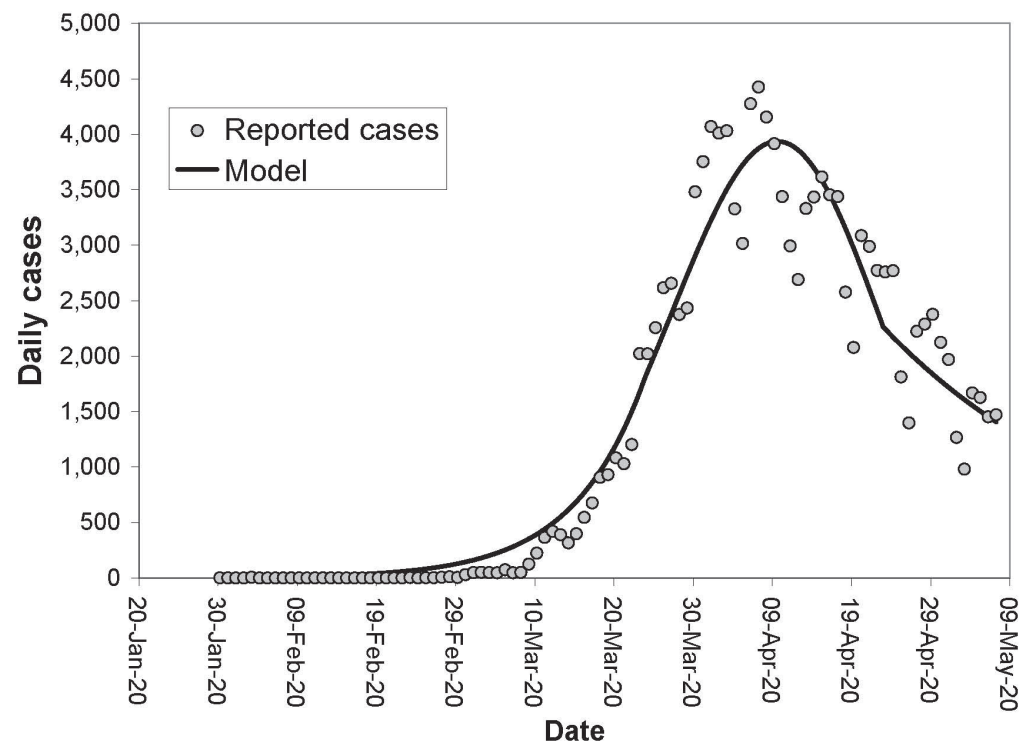

Figure 1. Comparison of model and daily new cases. The model has been matched to data up to 30 April 2020.

A dog leg can be seen in the fitted curve at 23 April 2020, corresponding to the point, 30 days after the start of lockdown, where the public's proficiency in social distancing is assumed to have stopped improving, as modelled by equation (1). From then on the daily new cases are predicted to continue falling in an exponential decline as a result of $\left(1-f_{\triangle R 0}\right) R_{0 i}(t)<1.0, i=1,2$.

The same feature is not immediately apparent in the data series. This may imply that the public is continuing to improve its observance of social distancing restrictions but it might mean that some other environmental effect is in play, such as the improving weather as winter turns into spring. If it is the latter, then the projections for the effect of lifting lockdown restrictions will be overly pessimistic for the summer period. On the other hand, one would then expect the situation to worsen significantly during the winter period.

\section{Modelling the exit from lockdown}

The first emergence from lockdown is assumed to take place over a period of 30 days from the date, $t_{R 1}$, when the first relaxation begins. Hence the basic reproduction numbers are given by 


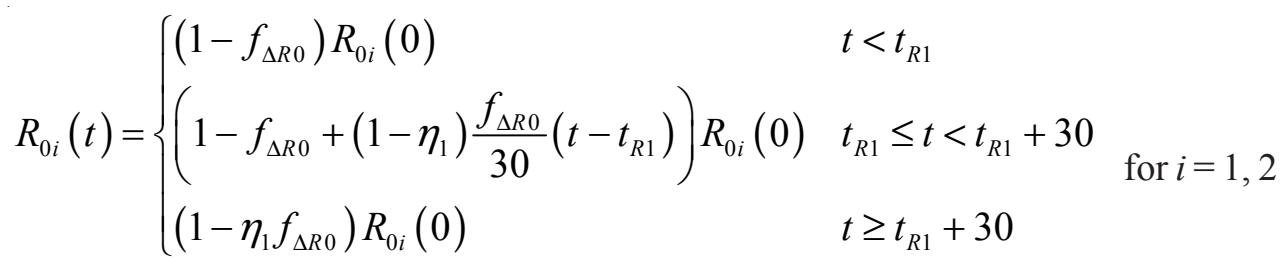

where the start date $t_{R 1}$ is 13 May 2020. Thus the first phase of leaving lockdown will be complete on 12 June 2020. Meanwhile, $\eta_{1}$ is the social distancing effectiveness of the first stage of emerging from lockdown. $\eta_{1}=1.0$ would keep all of the social distancing benefits of lockdown while $\eta_{1}=0.0$ would retain none. A derivation of equation (2) is given in Appendix A, Section A.3.

While it is possible to have just one stage of emergence from lockdown, this will not always be the case, and, indeed, the stages may be separated by up to a year or so. Allowance is made for this eventuality by modelling later stages of emergence by step functions. Hence, for the $k$ th stage of emergence:

$$
R_{0 i}(t)=\left\{\begin{array}{ll}
\left(1-\eta_{k-1} f_{\Delta R 0}\right) R_{0 i}(0) & t<t_{R k} \\
\left(1-\eta_{k} f_{\Delta R 0}\right) R_{0 i}(0) & t \geq t_{R k}
\end{array} \text { for } i=1,2\right.
$$

where $\eta_{k}$ is the social distancing effectiveness of the $k$ th stage of emerging from lockdown.

An overall basic reproduction number, $R_{0}(t)$, may be calculated as:

$$
R_{0}(t)=\theta_{1} R_{01}(t)+\theta_{2} R_{02}(t)
$$

The fraction of the population needing to have been infected to give population ("herd") immunity, above which the number of infectious people will decline, viz. $\mathrm{d} n / \mathrm{d} t<0$, is then: ${ }^{7}$

$$
\frac{n+n_{r}}{N}=1-\frac{1}{R_{0}(t)}
$$

where $n$ is the number of infectious people, $n_{r}$ is the number of people who have either recovered or else, unfortunately, have died and $N$ is the total number of people in the population. ${ }^{8}$

Alternatively, $R_{0}\left(t_{S}\right)$ may be "measured" by recording the computed values of $n$ and $n_{r}$ just before and just after the time, $t_{S}$, when $\mathrm{d} n\left(t_{S}\right) / \mathrm{d} t=0$ and then applying equation (5):

7 Equation (A.16) of ref. 3.

8 It should be noted that the rate of change in the number of infectious people is the difference between the rate at which people are becoming infected and the rate at which they are recovering (or dying). It will turn negative when fewer people are being infected than are recovering or dying, but people are still being infected. Hence the process of infection does not end at the point where equation (4) is satisfied. Thus the fraction of people infected by the end of an outbreak will always exceed the population immunity threshold for that value of $R_{0}$. The margin of exceedance will be small if population immunity has already been established through prior disease or by vaccination, but the overshoot can be sizeable in the case of an uncontrolled spread in a population with no prior immunity. A way of circumventing such an eventuality is to use social distancing to keep $R_{0}$ below its unconstrained value during the outbreak. Depending on the constrained value of $R_{0}$, the overshoot, which is inevitable, may not breach the unconstrained population immunity threshold by much, if at all. 


$$
R_{0}\left(t_{S}\right) \approx \frac{1}{2}\left(\frac{N}{N-\left(n\left(t_{S}^{-}\right)+n_{r}\left(t_{S}^{-}\right)\right)}+\frac{N}{N-\left(n\left(t_{S}^{+}\right)+n_{r}\left(t_{S}^{+}\right)\right)}\right)
$$

where the following two conditions hold: $t_{s}^{-}<t_{S}<t_{s}^{+}$and $t_{s}^{-} \approx t_{S} \approx t_{s}^{+}$, while, moreover, $\mathrm{d} n\left(t_{S}^{-}\right) / \mathrm{d} t>0$ and $\mathrm{d} n\left(t_{S}^{+}\right) / \mathrm{d} t<0$. The fraction of the population that needs to be infected to give population immunity may then be calculated using equation (5).

\section{Assessing the cost in human lives}

Two causes of loss of life are considered. Death can occur as a direct result of Covid-19, and the methods described in Thomas ${ }^{4}$ have been used to estimate the number of deaths from the disease. Since the average Covid-19 victim dying will lose 12 years of life expectancy, multiplying the number of victims by 12 gives the total life expectancy lost in years.

Reductions in life expectancy for the average person in the nation can also occur as result of national impoverishment. ${ }^{10,11}$ The Office of Budget Responsibility (OBR) has estimated the decline in growth for the 2 nd quarter of $2020^{12}$ would be $35 \%$ if the lockdown lasted 3 months. The OBR figures are used to calculate national output under the assumption that the weekly fractional change in output with respect to the average week's output in the first 12 weeks of 2020 is proportional to the social distancing effectiveness, as described in Appendix $\mathrm{C}$ of Thomas. ${ }^{4}$ This allows the annual GDP to be calculated and hence the GDP per head.

A method of calculating life expectancy when there is a long-term fall in GDP is given in Thomas, ${ }^{3,4}$ which makes use of the J-value explanation that there is a causal relationship between GDP per head and life expectancy. ${ }^{\mathbf{1 3}}$ The total loss of life expectancy in years may then be found by multiplying by the population of the UK, taken to be 67 million.

The loss of life in years may be converted into the number of average lives to come lost by dividing by the population-average life expectancy for 2020 . This is found from the life tables of the Office of National Statistics (ONS) ${ }^{\mathbf{1 4}}$ to be 41.8 years, which is rounded for convenience to 42 years.

The ONS ${ }^{15}$ has recorded an excess of non-Covid-19 deaths in the first half of 2020 , over and above the 5-year average. Some of these additional deaths might be due to unrecorded Covid-19,

9 Hanlon, P. et al. COVID-19 - exploring the implications of long-term condition type and extent of multimorbidity on years of life lost: a modelling study. Wellcome Open Research (2020) https:// wellcomeopenresearch.org/articles/5-75 [awaiting peer review].

${ }^{10}$ Thomas, P. and Waddington, I. Validating the J-value safety assessment tool against pan-national data. Process Safety Environmental Protection 112A (2017) 179-197.

11 Thomas, P. Corroboration of the J-value model for life-expectancy growth in industrialized countries. Nanotechnol. Perceptions 13 (2017) 31-44.

${ }^{12}$ Office of Budget Responsibility. Commentary on the OBR coronavirus reference scenario (14 April 2020) https://cdn.obr.uk/Coronavirus_reference_scenario_commentary.pdf

${ }^{13}$ When life expectancy is measured from birth, the relationship is known as the Preston curve. A more powerful result concerns the population-average life expectancy. ${ }^{\mathbf{1 0}}$

${ }^{14}$ Office of National Statistics, 2020, National life tables, UK: 2016 to 2018, https:/www.ons.gov.uk/ peoplepopulationandcommunity/birthsdeathsandmarriages/lifeexpectancies/bulletins/ nationallifetablesunitedkingdom/2016to2018

${ }^{15}$ Office of National Statistics, 2020, Deaths registered weekly in England and Wales, provisional: week 
but others might be the result of sick people not seeking the necessary medical treatment either because of the Government's general guidance to avoid hospitals and general practitioners' surgeries wherever possible or because of decisions they themselves made not to attend as a result of their own, probably incorrect, assessment of the relative risks. There were 6,767 additional nonCovid deaths over the 5-year average in England and Wales in the calendar year to 5 June 2020, $14 \%$ of the Covid-19 total. Loss of life from this indirect source appears to be relatively small when compared with that lost directly and has not been included in the analysis presented here.

\section{Results}

\section{Option 1: Keep the basic reproduction number, $\boldsymbol{R}_{\mathbf{0}}$, below 1.0}

There is limited room for relaxation given the effective value, 0.74 , of the combined reproduction number at the start of the process of leaving lockdown. $R_{0}(t)$ is assumed to rise to 0.999 by 12 June 2020 and then remain there, ensuring that near-lockdown conditions persist indefinitely into the future. The number of new daily cases of Covid-19 will continue to decline. Figure 2 shows the number of new daily cases normalized to the model's first peak of 3937 cases. Little immunity is built up: $5 \%$ of the population will have contracted the virus after several years, a long way below the $49 \%$ needed for population immunity. 16 Hence the nation will be almost as vulnerable to further epidemics in 2025 as it was in January 2020. National output drops to $71 \%$ of its prelockdown value and does not recover (Figure 3 ). While only a relatively small number of lives are lost to Covid-19, life expectancy declines due to serious national impoverishment.

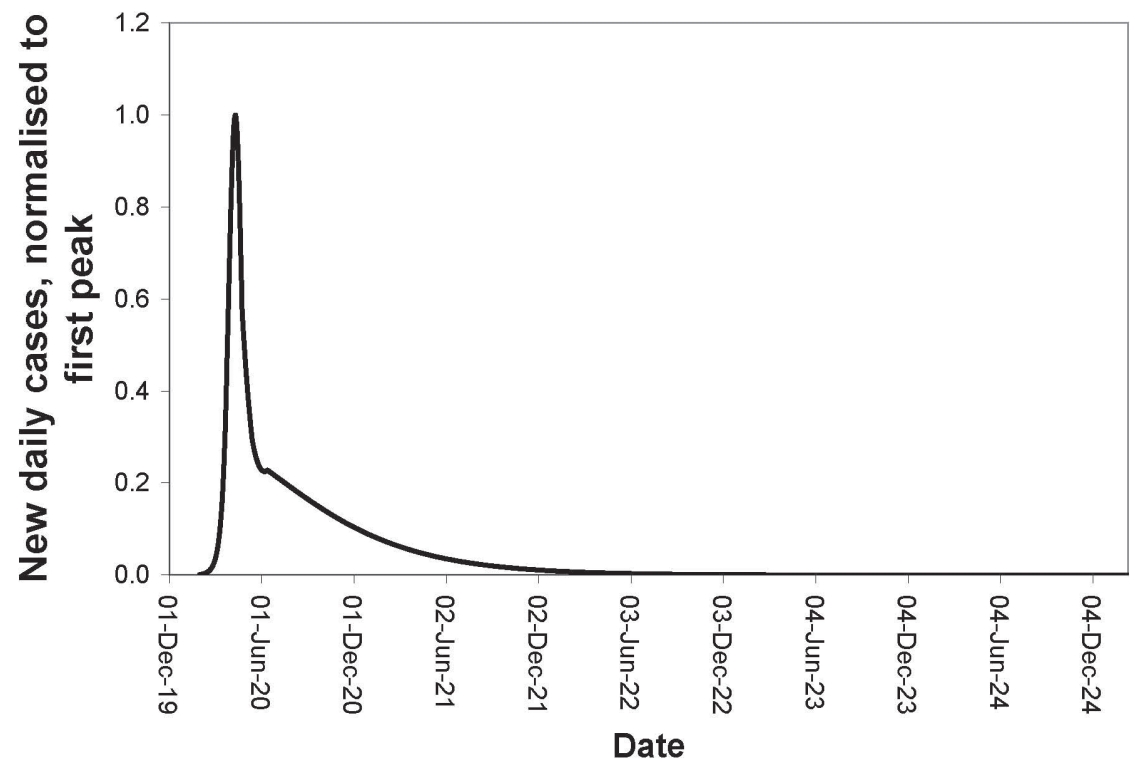

Figure 2. New daily cases normalised to the first peak, under Option 1: Keep the basic reproduction number, $R_{0}$, below 1.0 .

ending 5 June 2020, https://www.ons.gov.uk/peoplepopulationandcommunity/birthsdeathsandmarriages/ deaths/bulletins/deathsregisteredweeklyinenglandandwalesprovisional/latest 


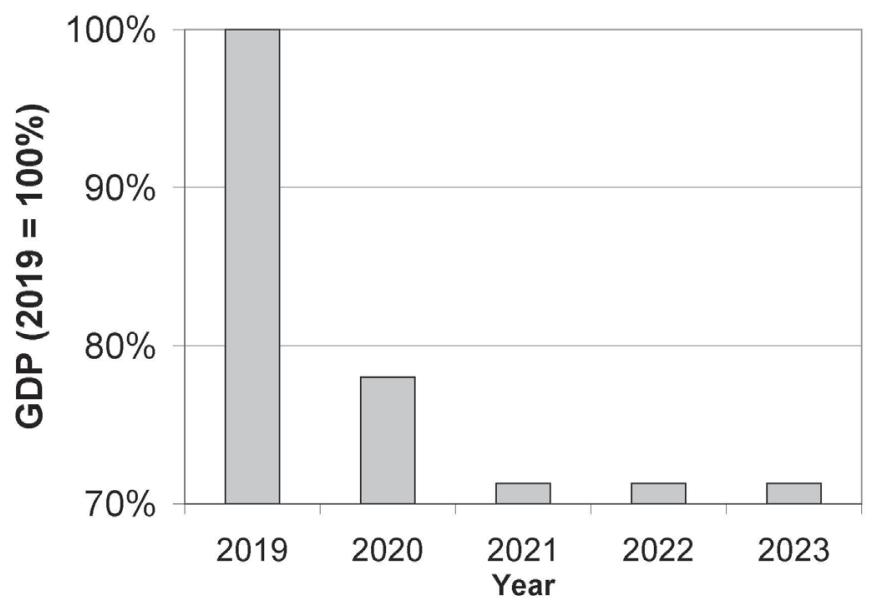

Figure 3. Annual GDP over time under Option 1: Keep the basic reproduction number, $R_{0}$, below 1.0.

The cost in human lives is $\sim 1.9$ million plex-2020, where 1 plex-2020 $=42$ years, the population-average life expectancy of a UK citizen in 2020. Almost all of these average UK lives are caused by the nation's fall in economic activity as a result of lockdown and continuing near-lockdown conditions (Table 2).

Table 2. Loss of life in plex-2020 under Option 1: Keep the basic reproduction number, $R_{0}$, below 1.0 .

\begin{tabular}{lc}
\hline Cause & Number of average lives lost (plex-2020) \\
\hline To Covid-19 & 6,000 \\
To national impoverishment & $1,863,000$ \\
Total & $1,869,000$ \\
\hline
\end{tabular}

\section{Option 2: Move out as quickly as possible without overstraining the health services}

Option 2 entails a slow move out of lockdown taken in steps, chosen to keep the peaks in new daily cases no higher than about twice that seen at the beginning of April 2020. The effective, combined basic reproduction number is allowed to rise to 1.18 by 12 June 2020 , and is held there steadily for about a year, when it is increased to 1.6. That value is held for a year, after which all restrictions are abolished, and $R_{0}(t)$ rises to its unconstrained level of 1.94 in June 2022 (Figure 4). The health services need to cope with two peaks each of which is twice the size of that seen in early April 2020. See Figure 5. This will, no doubt, require the Nightingale field hospitals to be brought back into service. The epidemic is essentially over by June 2022 . Half the population has been infected in the end, meaning that the nation has population immunity. ${ }^{16}$ Gross domestic product falls by $18.7 \%$ in 2020. The economy does not recover until 2023 (Figure 6).

\footnotetext{
${ }^{16}$ The figure for population ("herd") immunity was calculated by inserting the unconstrained value estimated for $R_{0}$, namely 1.94 , into equation (4): $1-1 / 1.94=0.485$.
} 


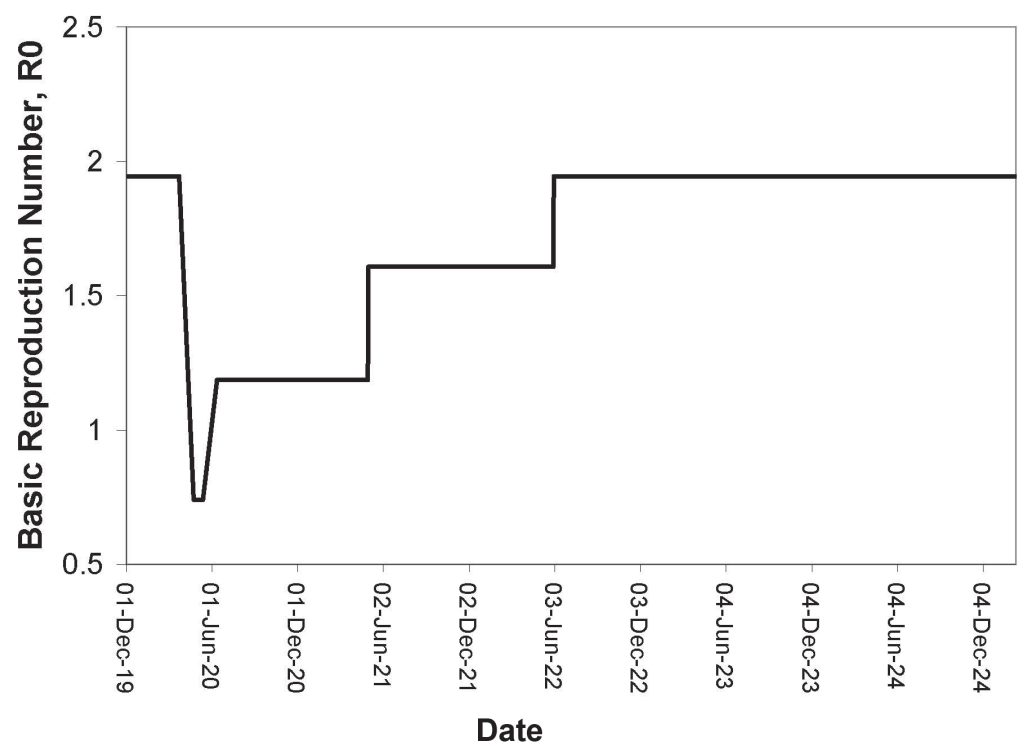

Figure 4. The trajectory of the effective combined reproduction number under Option 2: Move out as quickly as possible without overstraining the health services.

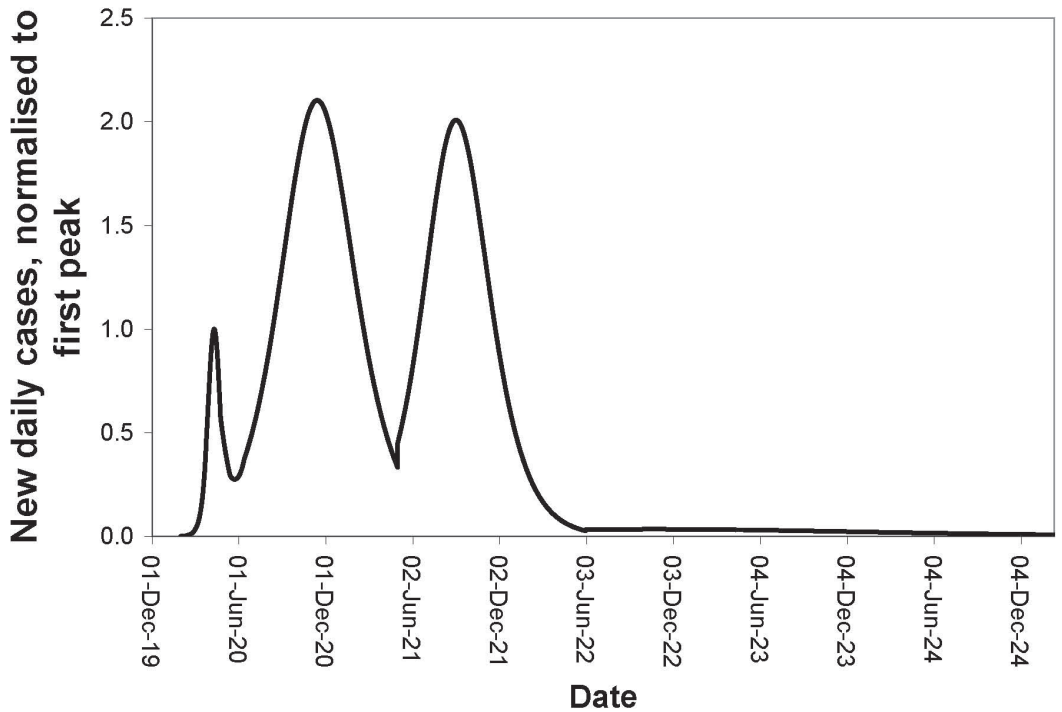

Figure 5. New daily cases under Option 2: Move out as quickly as possible without overstraining the health services. 


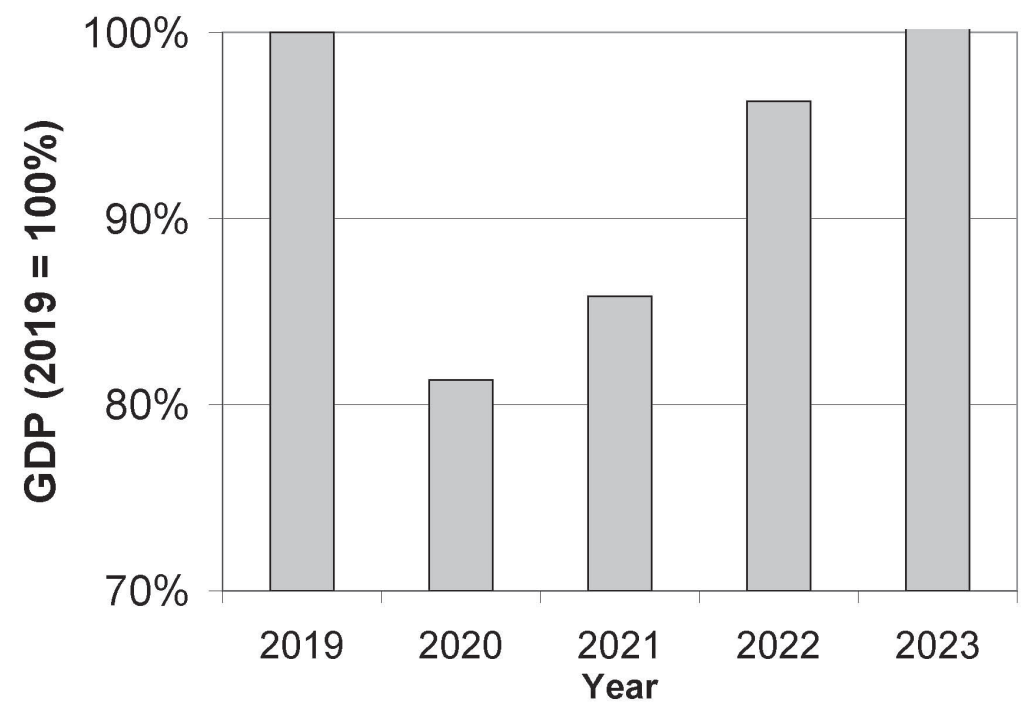

Figure 6. Annual GDP over time under Option 2: Move out as quickly as possible without overstraining the health services.

The cost in average human lives is 619,000 . Very roughly, $10 \%$ of this loss of life is caused by Covid-19 and $90 \%$ by the nation's fall in economic activity (Table 3 ).

Table 3. Loss of life in plex-2020 under Option 2: Move out as quickly as possible without overstraining the health services.

\begin{tabular}{lc}
\hline & Number of average lives lost (plex-2020) \\
\hline To Covid-19 & 55,000 \\
To national impoverishment & 564,000 \\
Total & 619,000 \\
\hline
\end{tabular}

\section{Option 3: Move out completely in 2020 while minimizing Covid-19 cases.}

The effective, combined basic reproduction number is allowed to rise to 1.45 by 12 June 2020 . All restrictions are removed on 1st January 2021, when $R_{0}(t)$ increases to its unconstrained level of 1.94 (Figure 7). There is a single additional wave, the peak of which occurs in September 2020 and is about 9 times the size of that seen in April 2020. (Figure 8). The health services would struggle to cope, and the death rate is assumed to double as a result. However, $54 \%$ of the population would be infected by the end of 2020 , so that population immunity would be developed. ${ }^{16}$ Gross domestic product falls by $14.6 \%$ in 2020 but the economy recovers in 2021 (Figure 9).

The number of average lives lost is 118,000 , due entirely to Covid- 19 under the assumption that few additional lives will be lost to national impoverishment because the recession is shortlived (Table 4). 


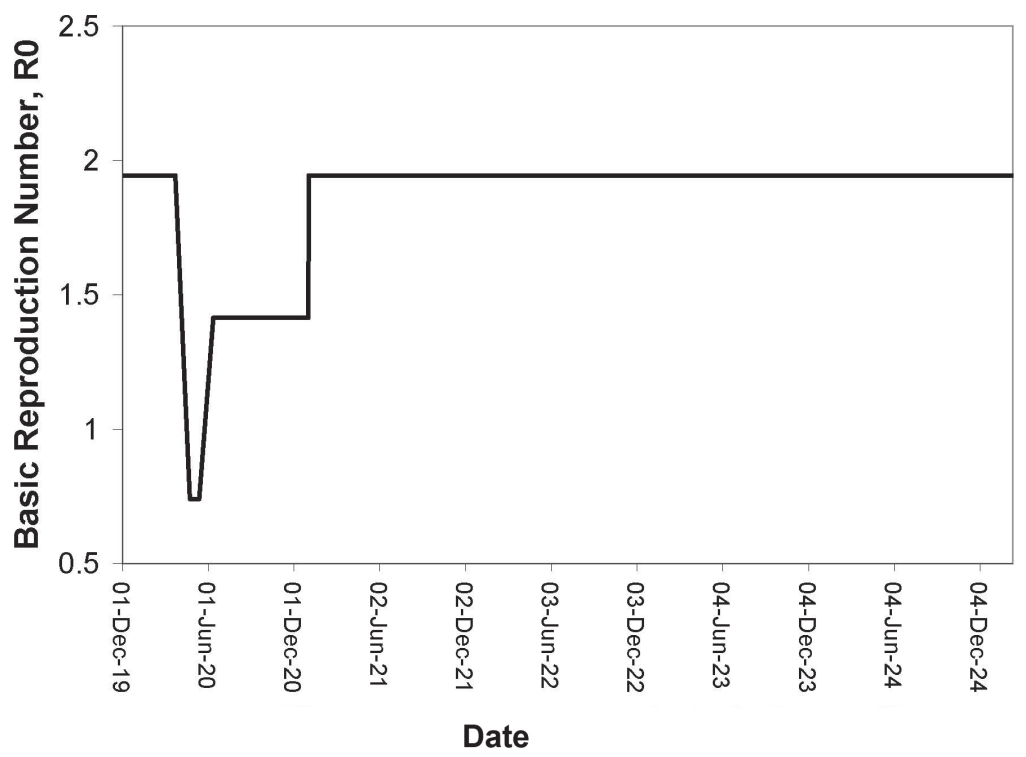

Figure 7. The trajectory of the effective combined reproduction number under Option 3: Move out completely in 2020 while minimizing Covid-19 cases.

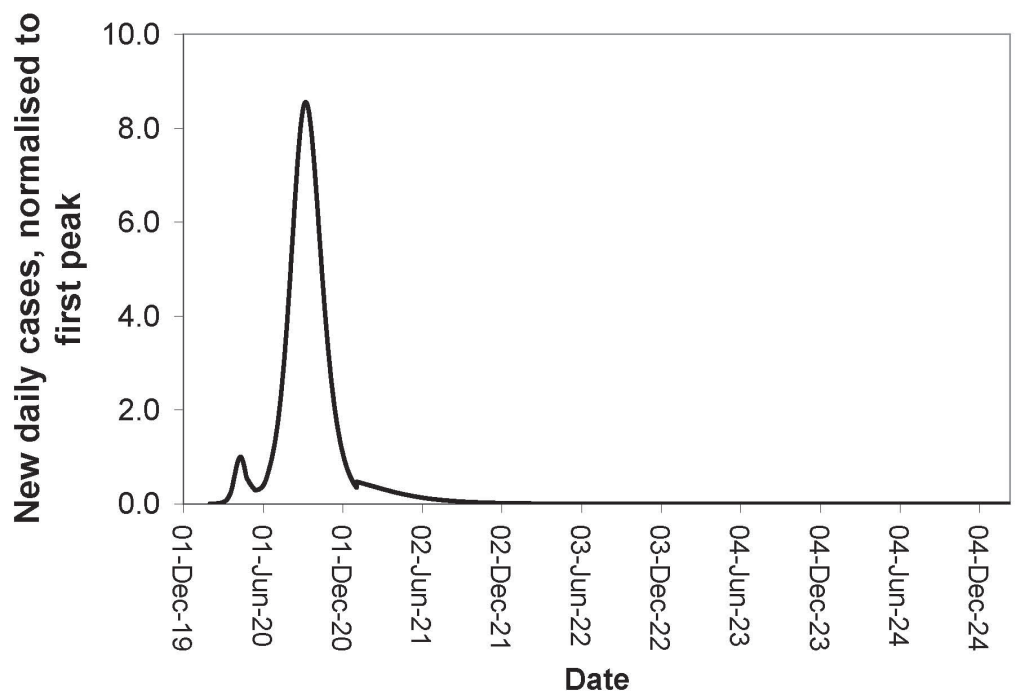

Figure 8. New daily cases under Option 3: Move out completely in 2020 while minimizing Covid-19 cases. 


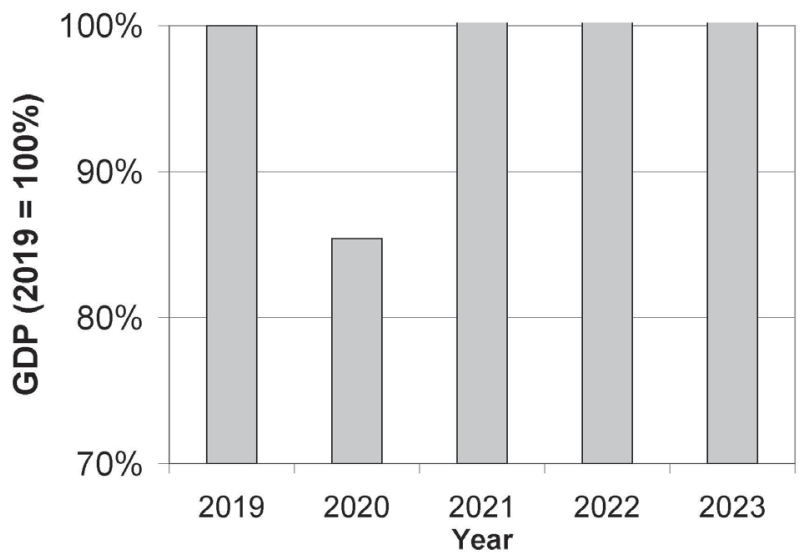

Figure 9. Annual GDP over time under Option 3: Move out completely in 2020 while minimizing Covid-19 cases.

Table 4. Loss of life in plex-2020 under Option 3: Move out completely in 2020 while minimizing Covid-19 cases.

\begin{tabular}{lc}
\hline & Number of average lives lost (plex-2020) \\
\hline To Covid-19 & 118,000 \\
To national impoverishment & Not applicable \\
Total & 118,000 \\
\hline
\end{tabular}

\section{Option 4: Move out of lockdown as fast as possible}

All restrictions are removed by 12 June 2020, when the effective, combined basic reproduction number returns to its unconstrained value of $R_{0}(t)=R_{0}(0)=1.94$. There is a single additional spike of infections peaking in late July 2020, 30 times the size of that seen in April 2020 (Figure 10). The health services can be expected to be overwhelmed, and the death rate is assumed to double as a result. $77 \%$ of the population are infected by the end of 2020 . Gross domestic product falls by $5.1 \%$ in 2020 but the economy recovers fully in 2021 (Figure 11).

The greater overshoot in infections (to $77 \%$ of the population) above the population immunity level $(\sim 49 \%)^{16}$ compared with Option 3, where only 54\% were calculated to have been infected, will mean that more people with die from Covid-19. The number of average lives lost is 170,000 . All are assumed to be caused by coronavirus as no lives will be lost to national impoverishment because the recession is very short lived. See Table 5.

\section{Sensitivity studies}

A feature of the previous research ${ }^{4}$ was the large number of possible sets of parameters able to fit the daily case data to 10 April 2020. However the extra data now available has imposed additional constraints through the need to match daily cases up to the later date of 30 April 2020 and to reproduce the survey estimate of the number of people outside the hospital system carrying an active infection within a week either side of 3 May 2020. This has narrowed the range of possible parameters. 


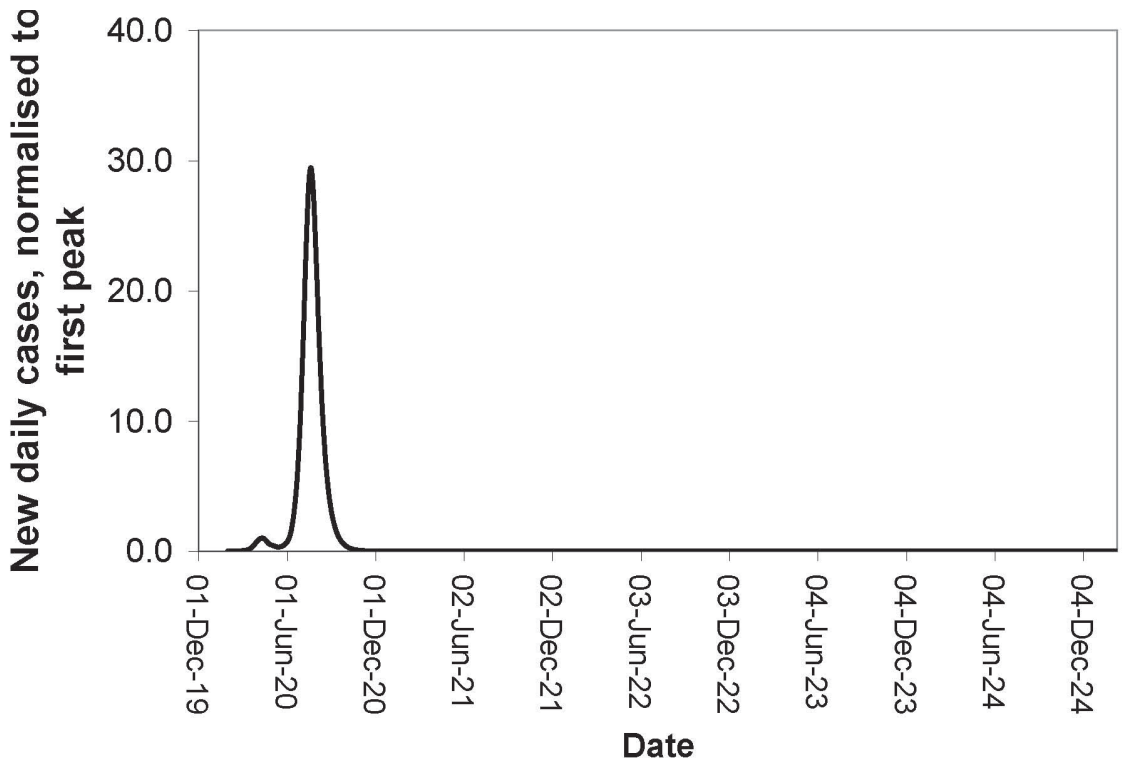

Figure 10. New daily cases under Option 4: Move out of lockdown as fast as possible.

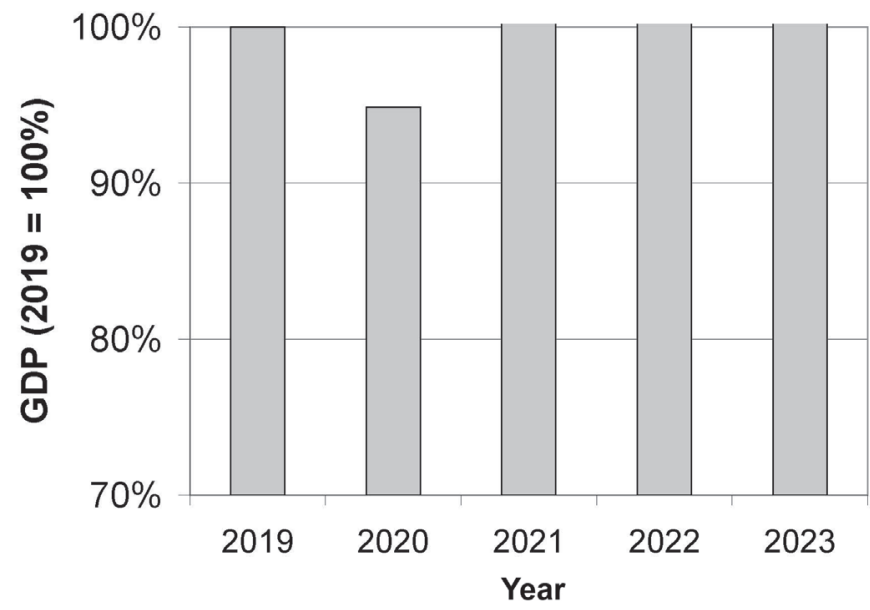

Figure 11. Annual GDP over time under Option 4: Move out of lockdown as fast as possible.

Table 5. Loss of life in plex-2020 under Option 4: Move out of lockdown as fast as possible.

\begin{tabular}{lc}
\hline & Number of average lives lost (plex-2020) \\
\hline To Covid-19 & 170,000 \\
To national impoverishment & Not applicable \\
Total & 170,000 \\
\hline
\end{tabular}


The sensitivity studies feature a new set of optimized parameters found from taking a different set of initial estimates from those that generated the data-set listed in Table 1 and used in Section 5. The new parameter set was found under the constraint that the average time from infection to passing on the infection was less than 12 days. See Table 6. Figure 12 shows the match between the daily new cases and the model prediction. The predicted curve has a very similar shape to that displayed in Figure 1, except that the dog leg is slightly sharper in the later figure.

Table 6. Epidemic parameter set for sensitivity studies of $\S 6$.

\begin{tabular}{lcc}
\hline & Cohort 1 & Cohort 2 \\
\hline Cohort index, $i$ & 1 & 2 \\
Fraction of population in cohort, $\theta_{i}$ & 0.134 & 0.866 \\
Basic reproduction number, $R_{0 i}$ & 2.67 & 1.940 \\
Average time between infections, $\tau_{\text {inf }, i}$ (days) & 10.78 & 9.22 \\
Number of people with active infections on 30 January 2020, $n_{i}(0)$ & 1 & 193 \\
\hline & Applicable to both cohorts \\
\hline Combined basic reproduction number before lockdown, $R_{0}$ & \multicolumn{2}{c}{2.04} \\
Population ("herd") immunity fraction & \multicolumn{2}{c}{$51 \%$} \\
Fractional decrease in the two basic reproduction numbers after 30 & \multicolumn{2}{c}{0.636} \\
$\quad$ days of lockdown, $f_{\Delta \mathrm{R} 0}$ & \multicolumn{2}{c}{0.74} \\
Combined basic reproduction number after 30 days of lockdown & \multicolumn{2}{c}{0.0066} \\
Overall fatality rate after infection & \multicolumn{2}{c}{387} \\
Root mean squared error between daily case numbers to 30 April 2020 & \multicolumn{2}{c}{} \\
\hline
\end{tabular}

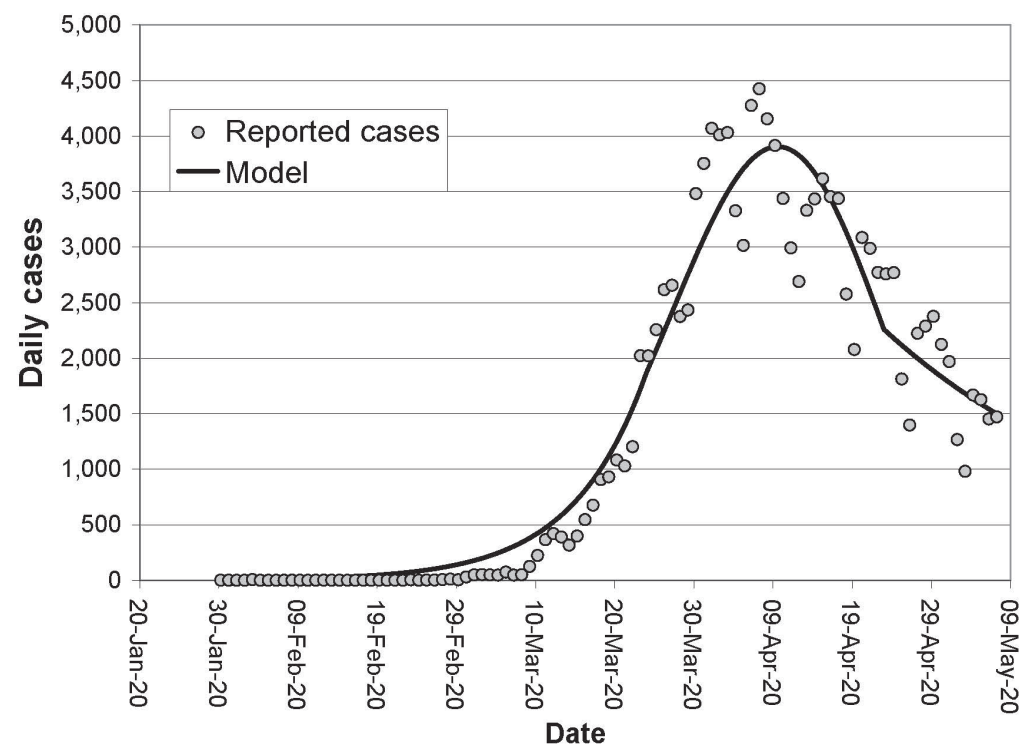

Figure 12. Model and daily new cases using the parameter set of the sensitivity studies. The model has been matched to data up to 30 April 2020. 


\subsection{Changes between the sensitivity studies and the base cases under Option 1: Keep the basic reproduction number, $R_{0}$, below 1.0}

There are relatively small changes between Option 1 (base case) and Option 1 (sensitivity study). The figure for total average lives lost increases from 1.869 million to 1.918 million plex-2020.

\subsection{Changes between the sensitivity studies and the base cases under Option 2: Move out as quickly as possible without overstraining the health services}

Compared with the same option in the base cases, the peaks of new daily cases in waves 2 and 3 go up from roughly twice to roughly two and a half times the maximum value observed at the beginning of April 2020 under Option 2 of the sensitivity studies. The total for average lives lost rises from 619,000 to 655,000 plex-2020.

If it is desired to keep the peaks of daily cases in waves 2 and 3 to no more than twice the peak experienced at the beginning of April 2020, then a smaller third wave is incurred. See Figure 13. A recession that is somewhat worse means that the total number of average lives lost rises to 719,000 plex-2020. ${ }^{17}$

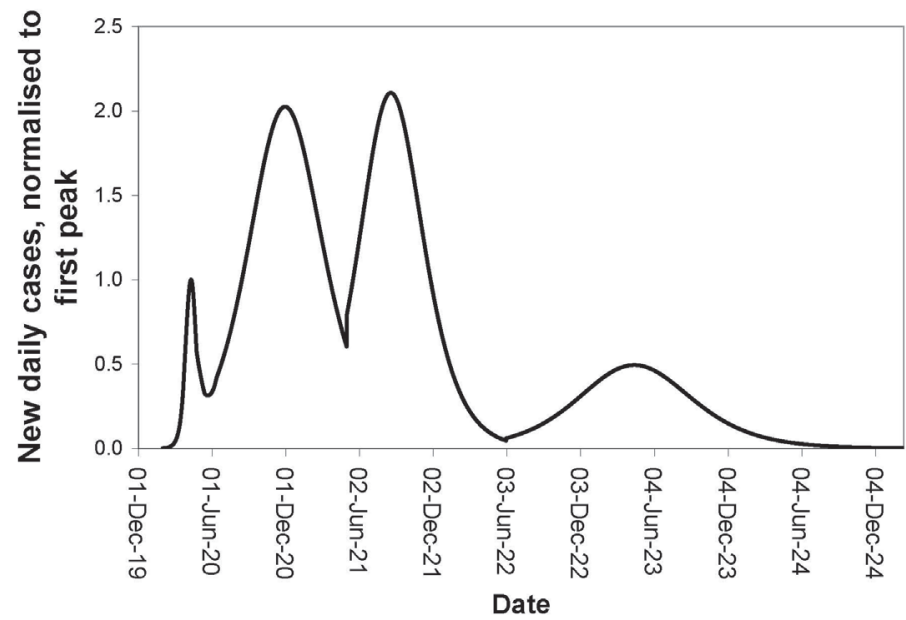

Figure 13. New daily cases under Option 2: Move out as quickly as possible without overstraining the health services; sensitivity studies with the constraint that the second and third peaks must be no greater than about twice the first peak.

${ }^{17}$ Recent information on the readiness of England's National Health Service suggests that NHS regions are planning for a second wave of infections that is larger than the first. For one of the seven such regions, the South East, the Health Service Journal states: ${ }^{18}$

Papers before clinical commissioning group governing bodies in Sussex say: 'Systems in the south east [have] started to coalesce around being prepared for a second wave of between 2 and where 2.5 [times] the first wave as a reasonable worst case scenario.'

the University of Sussex mathematics department has carried out modelling of three scenarios for the pandemic past the first peak, using R numbers of 1.1, 1.2 and 1.3, with 1.3 'cited as the reasonable worst case scenario by the military'.

${ }^{18}$ Moore, A. NHS planning for second covid peak more than double the size. Health Service J. (23 June 


\subsection{Changes between the sensitivity studies and the base cases under Option 3: Move out completely in 2020 while minimizing Covid-19 cases}

The responses are similar in these cases. Option 3 in the sensitivity studies calculates a somewhat higher total of average lives lost to the coronavirus: 148,000 compared with 118,000.

\subsection{Changes between the sensitivity studies and the base cases under Option 4: Move out of lockdown as fast as possible}

In the sensitivity studies, Option 4, the spike in daily new cases reaches a high point of 35 times the peak number found in April 2020, somewhat greater than the factor of 30 found in Option 4 of the base cases.

$80 \%$ rather than $77 \%$ of the population becomes infected, and this pushes the loss of life from 170,000 to 204,000 plex-2020.

\subsection{Overall comparison}

Table 7 compares the loss of life under all 4 options for the base cases and the sensitivity studies. The toll on life is consistently somewhat worse under each option in the sensitivity studies, but no difference is made in the ranking of the options.

Table 7. Loss of life (plex-2020) under the four options in the base cases and in the sensitivity studies

\begin{tabular}{lcc}
\hline & Base cases & Sensitivity studies \\
\hline $\begin{array}{l}\text { Option 1: Keep the basic reproduction } \\
\text { number, } R_{0} \text {, below 1.0 }\end{array}$ & $1,869,000$ & $1,918,000$ \\
$\begin{array}{l}\text { Option 2: Move out as quickly as possible } \\
\text { without overstraining the health services }\end{array}$ & 619,000 & 655,000 \\
$\begin{array}{l}\text { Option 3: Move out completely in 2020 } \\
\text { while minimizing Covid-19 cases }\end{array}$ & 118,000 & 148,000 \\
$\begin{array}{l}\text { Option 4: Move out of lockdown as fast as } \\
\text { possible }\end{array}$ & 170,000 & 204,000 \\
\hline
\end{tabular}

\section{Discussion}

The range of sets of parameters able to match the measured data on the coronavirus epidemic in the UK has narrowed as more empirical evidence has accumulated. This convergence increases confidence in the two-cohort model as a guide to future dynamic behaviour of the epidemic resulting from the easing of lockdown restrictions.

All options for leaving lockdown, except for Option 1, lead to a second wave of infections.

It is clear from Table 7 that a too rapid emergence from lockdown (Option 4) would precipitate an uncontrolled epidemic but that leaving rapidly (Option 3) would minimize loss of life.

Leaving lockdown whilst avoiding overstraining the health services (Option 2) might seem at first glance to be a desirable course of action, but the analysis presented here suggests that the

2020) https://www.hsj.co.uk/coronavirus/nhs-planning-for-second-covid-peak-more-than-doublethe-size/7027891.article?adredir=1 
deep and long recession provoked by such a slow departure would lead to great loss of life due to national impoverishment.

The adverse effects of national poverty are illustrated even more clearly in the case of Option 1, where the basic reproduction number and hence $R$-rate are kept below 1.0. This policy emerges as the worst in terms of loss of human life. The nation would face a huge and unprecedented setback to its economic well-being from which it would have little resource to recover. It is open to question how long Option 1 could be maintained in the face of social and political discontent that might mount.

The only way out for a nation embarking wholeheartedly on Option 1 would be the rapid discovery, test and manufacture and extensive deployment of an effective vaccine. Since the consequences of the option are extremely severe, adopting Option 1 would be akin to taking a gamble on such an eventuality. The average development time for a vaccine is 10.7 years. ${ }^{19}$ The development of the Ebola vaccine took 5-6 years, which was considered fast, while the record for the shortest development time is held by the mumps vaccine, which required 4 years. ${ }^{\mathbf{2 0}}$ Moreover, the probability of a vaccine being successfully developed from scratch to the point where it can be released for use is $6 \%{ }^{19}$

As of June 2020, there appeared to be four vaccines in Phase I trials, where the vaccine is given to small groups of humans: one in the UK, two in the USA and one in China. ${ }^{21}$ But the probability of going from Phase I trials to a successful launch may lie between $17 \%{ }^{19}$ and 39\%. ${ }^{22}$ Many vaccines against Covid-19 are under development across the world, but it is unwarrantedly optimistic to suppose that there is a high probability that a successful vaccine will be developed and deployed in the UK within the 4 year period considered in this study.

Option 3 emerges clearly as the best strategy for minimizing loss of life.

The possibility of unidentified environmental effects (e.g., summer weather) augmenting the reduction in infection transmission during the lockdown period was considered at the end of Section 2. If such effects existed and were seasonal, then benefit would be drawn from their presence in the spring and summer. This would make a fuller exit from lockdown easier. But the beneficial effect would then be negated in late autumn and winter.

On the other hand, if the downward pressure on infection transmission were due to a permanently present mechanism, for example, cross-immunity, ${ }^{23}$ then the size of the 2 nd wave predicted for Option 3 would be reduced, further increasing the attractions of that option.

${ }^{19}$ Pronker, E.S, Weenen, T.C., Commandeur, H., Claassen, E.H.J.H.M., Osterhaus, A.D.M.E., 2013,) Risk in Vaccine Research and Development Quantified. PLOS ONE 8(3): e57755. doi:10.1371/ journal.pone.0057755, https://www.ncbi.nlm.nih.gov/pmc/articles/PMC3603987/pdf/pone.0057755.pdf

${ }^{20}$ Altman, M. J., 2020, Q\&A with Dr. Peter Hotez: Behind the Scenes of COVID-19 Vaccine Research, United Nations Foundation, 15 May, https://unfoundation.org/blog/post/qa-with-dr-peter-hotezbehind-the-scenes-of-covid-19-vaccine-research/

${ }^{21}$ Gartner, A., Roberts, L. and Hope, C., 2020, How close are we to a coronavirus vaccine? Latest news on UK and US trials, The Telegraph, 20 June 2020, https://www.telegraph.co.uk/global-health/ science-and-disease/covid-19-vaccine-trials-coronavirus-uk-human-treatment-latest/

22 Struck, M.-M. Vaccine R\&D success rates and development times. Nature Biotechnol. 14 (1996) 591-593.

${ }^{23}$ Gomez, M. G. M., et al., 2020, Individual variation in susceptibility or exposure to SARS-CoV-2 lowers the herd immunity threshold, medRxiv preprint doi: https://doi.org/10.1101/2020.04.27.20081893 


\section{Conclusions}

In the absence of a vaccine available in the near future, the exit from lockdown ought to be made rapidly if loss of life is to be minimized. That exit should, however, be controlled to avoid too great a spike in infections.

Option 3 minimizes overall loss of life, suggesting that attempts should be made to approach this strategy as closely as possible. This makes it advisable to extend health services capacity for Covid-19 cases further in anticipation of a second wave that could be substantially more than double the size of the first.

A second wave is an inevitable effect of all leaving strategies except for Option 1. This option, where the basic reproduction number and hence $R$-rate are kept below 1.0, would cause the greatest national impoverishment and the highest loss of life as a result.

While the model has been matched to UK epidemiological and economic conditions, its general findings are likely to be relevant to other developed countries in addition to the United Kingdom.

\section{Appendix A. Social distancing effectiveness}

\section{A.1 Locking down: derivation of equation (1) in the main text}

Suppose that the process of lockdown begins at time, $t_{L}$, and is completed at a later time, $t_{L C}$. The reproduction number at the start of the process, $R_{0 i}\left(t_{L}\right)$, will be unchanged from its original value:

$$
R_{0 i}\left(t_{L}\right)=R_{0 i}(0)
$$

but when the process of lockdown is complete, the basic reproduction number will have fallen to $R_{0 i}\left(t_{L C}\right)$. Assuming that the lockdown process is linear over time, then

$$
\frac{R_{0 i}(t)-R_{0 i}\left(t_{L}\right)}{R_{0 i}\left(t_{L C}\right)-R_{0 i}\left(t_{L}\right)}=\frac{t-t_{L}}{t_{L C}-t_{L}} \quad t_{L} \leq t \leq t_{L C}
$$

Rearranging and using equation (A.1):

$$
R_{0 i}(t)=R_{0 i}(0)+\frac{t-t_{L}}{t_{L C}-t_{L}}\left(R_{0 i}\left(t_{L C}\right)-R_{0 i}(0)\right) \quad t_{L} \leq t \leq t_{L C} .
$$

Meanwhile the fractional decrease, $f_{\Delta R 0}$, in the basic reproduction number between its initial value, $R_{0 i}(0)$, and its lockdown value, $R_{0 i}\left(t_{L C}\right)$ may be written:

$$
f_{\Delta R 0}=\frac{R_{0 i}(0)-R_{0 i}\left(t_{L C}\right)}{R_{0 i}(0)} .
$$

Substituting from equation (A.4) into equation (A.3) gives:

$$
R_{0 i}(t)=\left(1-\frac{t-t_{L}}{t_{L C}-t_{L}} f_{\Delta R 0}\right) R_{0 i}(0) \quad t_{L} \leq t \leq t_{L C} .
$$

The assumption is made in the main text that

$$
t_{L C}-t_{L}=30 \text { days. }
$$

Equations (A.5) and (A.6) then provide the basis for equation (1) in the main text. 


\section{A.2 Defining social distancing effectiveness}

At any time, $t$, between the start and completion of lockdown, the change, $\Delta R_{0 i}(t)$, in the basic reproduction number will be:

$$
\Delta R_{0 i}(t)=R_{0 i}\left(t_{L}\right)-R_{0 i}(t) \quad t_{L} \leq t \leq t_{L C} .
$$

Thus at the time, $t_{L C}$, when lockdown has just been completed:

$$
\Delta R_{0 i}\left(t_{L C}\right)=R_{0 i}\left(t_{L}\right)-R_{0 i}\left(t_{L C}\right) .
$$

It is natural to assume that the effectiveness of social distancing reaches a maximum at this point of complete lockdown. This implies that $\Delta R_{0 i}\left(t_{L C}\right)=\max \Delta R_{0 i}(t)$.

Given this maximum value for $\Delta R_{0 i}(t)$, it possible to define a "social distancing effectiveness", $\eta(t)$, obeying: $0 \leq \eta(t) \leq 1.0$, as the fraction of the maximum change in the reproduction rate:

$$
\eta(t)=\frac{\Delta R_{0 i}(t)}{\Delta R_{0 i}\left(t_{L C}\right)} .
$$

The right hand side of equation (A.9) may be found by dividing equation (A.7) by equation (A.8):

$$
\eta(t)=\frac{R_{0 i}\left(t_{L}\right)-R_{0 i}(t)}{R_{0 i}\left(t_{L}\right)-R_{0 i}\left(t_{L C}\right)}=\frac{R_{0 i}(0)-R_{0 i}(t)}{R_{0 i}(0)-R_{0 i}\left(t_{L C}\right)}
$$

where the second step follows from equation (A.1).

Social distancing effectiveness, as defined by Equation (A.10), may be used to characterize all situations between no social distancing measures being applied and full lockdown, irrespective of whether the degree of lockdown is being tightened or relaxed.

\section{A.3 1st relaxation of lockdown: derivation of equation (2) in the main text}

The basic reproduction number at the time, $t_{R 1}$, that the first relaxation begins will be that pertaining at the completion of lockdown, viz. $R_{0 i}\left(t_{R 1}\right)=R_{0 i}\left(t_{L C}\right)$. When the first relaxation is complete at time, $t_{R 1 C}$, the basic reproduction number will have risen to $R_{0 i}\left(t_{R 1 C}\right)$.

Assume the relaxation process is linear over time, so that

$$
\frac{R_{0 i}(t)-R_{0 i}\left(t_{R 1}\right)}{R_{0 i}\left(t_{R 1 C}\right)-R_{0 i}\left(t_{R 1}\right)}=\frac{t-t_{R 1}}{t_{R 1 C}-t_{R 1}} \quad t_{R 1} \leq t \leq t_{R 1 C} .
$$

Rearranging and noting that the basic reproduction number at the start of relaxation is the same as it was at the completion of lockdown, viz. $R_{0 i}\left(t_{R 1}\right)=R_{0 i}\left(t_{L C}\right)$, allows equation (A.11) to be written as

$$
R_{0 i}(t)=R_{0 i}\left(t_{L C}\right)+\frac{t-t_{R 1}}{t_{R 1 C}-t_{R 1}}\left(R_{0 i}\left(t_{R 1 C}\right)-R_{0 i}\left(t_{L C}\right)\right) \quad t_{R} \leq t \leq t_{R C} .
$$

Meanwhile, setting $t$ equal to $t_{R 1 C}$ in equation (A.10) gives

$$
\eta\left(t_{R 1 C}\right)=\frac{\Delta R_{0 i}\left(t_{R 1 C}\right)}{\Delta R_{0 i}\left(t_{L C}\right)}=\frac{R_{0 i}(0)-R_{0 i}\left(t_{R 1 C}\right)}{R_{0 i}(0)-R_{0 i}\left(t_{L C}\right)} .
$$


Hence the basic reproduction number at the completion of relaxation will be:

$$
R_{0 i}\left(t_{R 1 C}\right)=R_{0 i}(0)-\eta\left(t_{R 1 C}\right)\left(R_{0 i}(0)-R_{0 i}\left(t_{L C}\right)\right) .
$$

Meanwhile putting $t=t_{R 1 C}$ in equation (A.5) gives:

$$
R_{0 i}\left(t_{L C}\right)=R_{0 i}(0)\left(1-f_{\triangle R 0}\right) .
$$

Substituting from equation (A.15) into equation (A.14) yields

$$
R_{0 i}\left(t_{R 1 C}\right)=\left(1-\eta\left(t_{R 1 C}\right) f_{\triangle R 0}\right) R_{0 i}(0) \text {. }
$$

Substituting from equations (A.15) and (A.16) into equation (A.12) gives the following expression for the basic reproduction number at time, $t$, during the process of 1 st relaxation:

$$
R_{0 i}(t)=\left(1-f_{\triangle R 0}+\frac{t-t_{R 1}}{t_{R 1 C}-t_{R 1}}\left(1-\eta\left(t_{R 1 C}\right)\right) f_{\triangle R 0}\right) R_{0 i}(0) \quad t_{R} \leq t \leq t_{R C}
$$

Equation (A.17) can be seen to be equivalent to equation (2) in the main text after putting $\eta_{1}=\eta\left(t_{R 1 C}\right)$, and $t_{R 1 C}-t_{R 1}=30$, with the latter implying that it takes 30 days to move out of lockdown. 